\title{
Theoretical Considerations on Combined Optical Distance Measurements Using a Femtosecond Pulse Laser
}

\author{
Ki-Nam $\mathrm{Joo}^{1,2 *}$ and Seung-Woo $\mathrm{Kim}^{2}$ \\ ${ }^{1}$ Department of Photonics Engineering, Chosun University, \\ 375 Seosuk-dong, Dong-gu, Gwanju 501-759, Korea \\ ${ }^{2}$ Department of Mechanical Engineering, Korea Advanced Institute of Science and Technology, \\ Science Town, Daejeon 305-701, Korea
}

(Received July 25, 2012 : revised August 20, 2012 : accepted September 26, 2012)

\begin{abstract}
We introduce a combined technique and the mathematical description for distance measurements using a femtosecond pulse laser in a long range and a fine resolution. For distance measurements, the maximum measurable range can be extended by combining measurement results from several different methods while requiring relationships between the different measurement uncertainties and unambiguity ranges. This paper briefly explains why the uncertainty of a rough measurement technique (RMT) should be, at least, smaller than the half unambiguity range of a fine measurement technique (FMT) in order to combine a FMT with a RMT. Further discussions about the total measurement range, resolution, and uncertainty for various optical measurement techniques are also discussed.
\end{abstract}

Keywords: Combined optical interferometry, Distance measurements, Femtosecond pulse laser application OCIS codes : (120.3180) Interferometry; (120.0120) Instrumentation; measurement and metrology; (140.7090) Ultrafast lasers

\section{INTRODUCTION}

Optical interferometry for measuring displacements and distances has been a key technology in both industrial and scientific fields which need highly accurate positioning. However, typical homodyne/heterodyne interferometry can measure displacements and it has the limitation for measuring absolute distance measurements due to its short unambiguity range determined by the used wavelength. To avoid the ambiguity problem in displacement interferometry, various research including synthetic wavelength interferometry [1], a frequency modulated continuous wave method [2], multiple wavelength interferometry [3], and frequency sweeping interferometry [4] have been applied to extend the unambiguity range. Recently, the application of a femtosecond pulse laser for distance metrology not only offers further improvements but also makes it possible to measure absolute distances precisely from industrial applications to space programs [5-11].

The fundamental rule for distance measurements is that the measurement uncertainty and resolution are strongly related to the measurement range, i.e. an unambiguity range. For example, measurement techniques such as synthetic wavelength interferometers have relatively long unambiguity ranges (a few millimeters) but they can only measure distances with low resolutions compared to the conventional displacement interferometer. Then, for fine resolution and accurate measurements, the unambiguity range should be relatively short. In this case, however, the ambiguity problem can limit the maximum measurable range as explained in displacement interferometry. The measurement uncertainty also has similar aspects to measurement resolutions. In order to enhance the dynamic range, i.e. to measure distances with high resolution over a long range, combining measurement results from several methods with different resolutions and unambiguity ranges can be effective and can result in a good measurement performance $[5,9,12]$. For combining measurement results and successfully determining the distance value, however, the relationship between measured distances which have

\footnotetext{
*Corresponding author: knjoo@chosun.ac.kr

Color versions of one or more of the figures in this paper are available online.
} 
different uncertainties, resolutions, and unambiguity ranges should be taken into account [13]. In this paper, the principle of combined interferometry using a femtosecond pulse laser is explained and the practical conditions are derived with mathematical descriptions. Some considerations on the total measurement range, resolution, and uncertainty are also discussed.

\section{CONSIDERATIONS OF COMBINED OPTICAL DISTANCE MEASUREMENTS}

To describe the principle of combined interferometry, at least two measurement results which have well-defined unambiguity ranges, resolutions and measurement uncertainties are needed. For this discussion, a rough measurement technique (RMT) is defined as the method, which has a relatively poor resolution and a long unambiguity range. On the other hand, a fine measurement technique (FMT) is set as the method with a high resolution and its unambiguity range is small. These two techniques are simultaneously used to measure the same distance. In this case, the multiple integer order $(m)$ for the unambiguity range of the FMT is

$$
m=I N T\left[\frac{L}{l_{u}}\right]
$$

where $L$ is the measured distance from the RMT and $l_{u}$ is the unambiguity range of the FMT. The function $I N T[A]$ gives the integer part of $A$. Then, the combined measured distance $(D)$ is calculated as

$$
D=m \cdot l_{u}+l
$$

where $l$ is the measured distance from the FMT. Fig. 1(a) shows the operation principle of the combined method with the RMT and the FMT. This combined measurement can be accomplished in the range of the unambiguity range of the RMT, i.e. the maximum measurable range of the combined system, with the measurement resolution of the FMT. As the result, the dynamic range can be higher than those of the original techniques.

A typical example is the RF synthetic wavelength interferometry using the harmonics of repetition frequency (mode spacing frequency) in a femtosecond pulse laser. Assuming the repetition frequency of the femtosecond pulse laser is $75 \mathrm{MHz}$, the synthetic wavelength and the unambiguity range caused by this frequency in the Michelson type interferometers become $4.0 \mathrm{~m}$ and $2.0 \mathrm{~m}$, respectively. The $10^{\text {th }}$ harmonic of the fundamental frequency is $750 \mathrm{MHz}$, which means the synthetic wavelength and the unambiguity range become 10 times smaller than the original ones. If these two measurement results are successfully combined, the measurement resolution can be $0.056 \mathrm{~mm}$

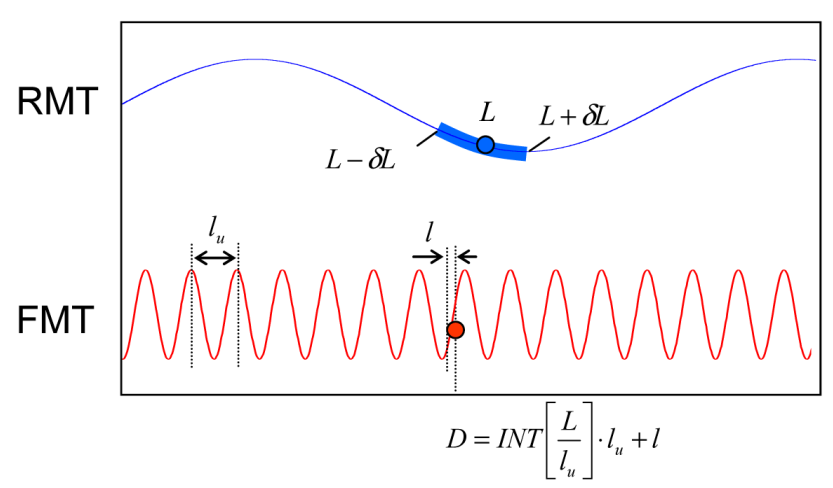

(a)

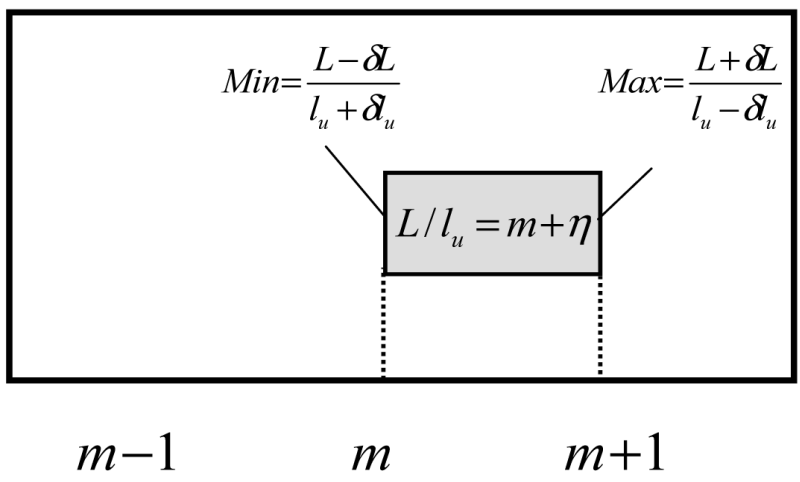

(b)

FIG. 1. (a) Operation principle of combined method using a rough measurement and a fine measurement techniques (RMT and FMT), (b) schematic of the conditions to determine the proper $\mathrm{m}$ value using Eq. (1). The ratio including measurement uncertainties should be larger than $m$ and smaller than $m+1$.

with a $2.0 \mathrm{~m}$ range assuming the electronic phase resolution is $0.1^{\circ}$. Combining these two techniques can enhance the dynamic range by a factor of 10 (in this example) while the original techniques have a dynamic range driven by the resolution of the phase meter.

The most important consideration in Eq. (2) is properly determining $m$ because an error in the integer order can lead to distance errors several orders higher than $l_{u}$. The primary condition for using combined techniques is the resolution of the RMT should be much smaller than $l_{u}$. However, this condition is not sufficient because each measurement quantity has its own measurement uncertainty. The function $I N T\left[L / l_{u}\right]$ only gives the integer part from $L / l_{u}$, so the measurement uncertainties from $L(\delta L)$ and $l_{u}$ $\left(\delta l_{u}\right)$ can induce errors in accurately determining the integer. To consider these measurement uncertainties in a combined technique, $L / l_{u}$ can be described with the integer and fraction $(\eta)$ parts as

$$
\frac{L}{l_{u}}=m+\eta
$$


The ratio $\left(L / l_{u}\right)$ has a maximum $($ Max $)$ and a minimum (Min) values, including measurement uncertainties from Eq. (3), of

$$
\begin{aligned}
& \operatorname{Max}=\frac{L+\delta L}{l_{u}-\delta l_{u}}=(m+\eta) \frac{1+\alpha}{1-\beta} \\
& \operatorname{Min}=\frac{L-\delta L}{l_{u}+\delta l_{u}}=(m+\eta) \frac{1-\alpha}{1+\beta}
\end{aligned}
$$

where $\alpha$ and $\beta$ are defined as the relative uncertainties $\delta$ $L / L$ and $\delta l_{u} / l_{u}$, respectively. In order to determine the accurate $m$ using Eq. (1), Max should be smaller than $m+1$ and Min should not be smaller than $m$ as shown in Fig. 1(b).

$$
\begin{aligned}
& \operatorname{Max}<m+1 \\
& \operatorname{Min} \geq m
\end{aligned}
$$

To simplify Eq. (4)-(6) further, two special cases are considered

\section{(1) Case 1: $a=\beta<<1$}

When the relative measurement uncertainty of the RMT $(\alpha)$ is same as the stability of unambiguity range in the FMT $(\beta)$, the condition for determining the right $m$ value can be simplified by substituting Eq. (4) and (5) into Eq. (6) where

$$
\begin{aligned}
& \alpha<\frac{(1-\eta)}{(2 m+\eta+1)} \\
& \alpha \leq \frac{\eta}{2(m+\eta)}
\end{aligned}
$$

According to Eq. (7) and (8), a large $m$ value for the long distance measurement is limited by $\alpha$, the uncertainties of the RMT and $l_{u}$. Therefore, determining $m$ using Eq. (1) can give errors beyond the upper conditions although the resolution of the RMT is much smaller than $l_{u}$. The other remarkable parameter is the fraction part $(\eta)$ of $\left(L / l_{u}\right)$. As $\eta$ approaches 0 or 1 , the right sides in Eq. (7), (8) approach 0 . These situations create a case where a finite $a$ must be close to zero, which is not possible, meaning the uncertainty criteria cannot be satisfied. For example, assume $L, l_{u}$ and $l$ are $40.79 \mathrm{~mm}, 5.1 \mathrm{~mm}$ and $0.2135 \mathrm{~mm}$, respectively. The integer, $m$, and the fraction, $\eta$, are 7 and 0.998 , respectively (from Eq. (1)) and then the total distance, $D$, becomes $7 \times 5.1+0.2135=35.9135$ $\mathrm{mm}$. If the real distance is $41 \mathrm{~mm}$, the measurement error is $5.0865 \mathrm{~mm}$, which is essentially the same as $l_{u}$.

\section{(2) Case 2: $\beta<<\alpha<<1$}

In most of measurements, the stability of the unambiguity range is very high because of the stabilization methods, which is a more realistic representation of the actual system. (The typical wavelength stability is better than $10^{-9}$ in typical interferometer applications.) Under this assumption, the conditions from Eq. (4)-(6) are

$$
\begin{aligned}
& \alpha<\frac{(1-\eta)}{(m+\eta)} \\
& \alpha \leq \frac{\eta}{(m+\eta)}
\end{aligned}
$$

Although Eq. (9) and (10) are twice the values from Eq. (7) and (8), as was the situation in Case 1, the same criteria for Case 1 exists for Case 2. For a successful operation of the combined technique, $m$ needs to be limited by the measurement uncertainty of RMT and $\eta$ should not be close to 0 or 1 as shown in both cases.

However, it is possible to determine $m$ accurately by comparing the measurement result and uncertainty in the RMT, even though $\eta$ is close to 0 or 1 . Because the total distance, $D$, should be in the RMT uncertainty range, the value is calculated using Eq. (2) and it can then be used to restrict the value for $m$. The calculated $D$ should be in the range of

$$
L-\delta L \leq D \leq L+\delta L
$$

If $m$ is not calculated correctly, the inequality of Eq. (11) is not satisfied and the alternatives $(m+1$ or $m-1)$ can be selected. Ambiguity of the selected value for $m$ in Eq. (11) can be avoided by ensuring $\delta L$ is smaller than $l_{u} / 2$. This relationship can be simplified as

$$
\alpha=\frac{\delta L}{L}<\frac{l_{u}}{2 L} \cong \frac{1}{2 m}
$$

In the previous example, the calculated distance, 35.9135 $\mathrm{mm}$ is out of range based on Eq. (11) if $\delta L$ is $0.5 \mathrm{~mm}(\delta$ $\left.L<l_{u} / 2\right)$. Then, the interger multiple can be increased or decreased by one and then rechecked to validate Eq. (11). In this case, the multiple integer can be selected as 8 $(m+1)$ and the measured distance, $D$, becomes $41.0135 \mathrm{~mm}$. The result is within the possible range from Eq. (12), thus, this is the final distance value from the combined measurement technique.

To summarize, the procedure for combining distance measurement techniques is as follows. First, the unambiguity range, resolution, and uncertainty for each measurement technique should be defined and the distances from each technique are measured simultaneously. Second, the multiple orders of the unambiguity range in the FMT are calculated using Eq. (1). Finally, the calculated distance is verified with Eq. (12). If the measured result fails this check, the integer should be increased or decreased by one and rechecked. For long distance measurements, the measurement uncertainty of the RMT should be enhanced according to Eq. (12). 


\section{EXPERIMENT AND DISCUSSION}

Figure 2 shows a combined technique using synthetic wavelength interferometry for the RMT and a spectrallyresolved interferometer for the FMT compared with a displacement interferometer (5510A, Agilent). The combined measurement adopted a femtosecond pulse laser as the source for both techniques simultaneously and the distance measurements had steps of $0.5 \mathrm{~mm}$ with a $50 \mathrm{~mm}$ range. The synthetic wavelength interferometer measured distances

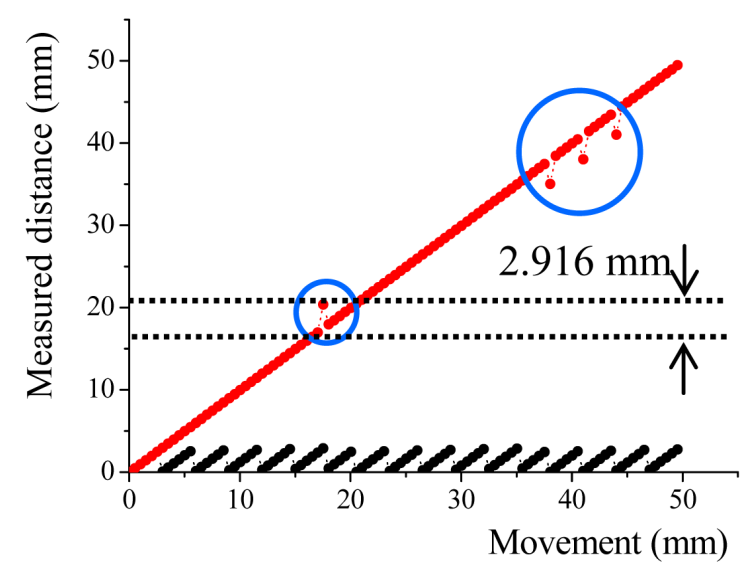

(a)

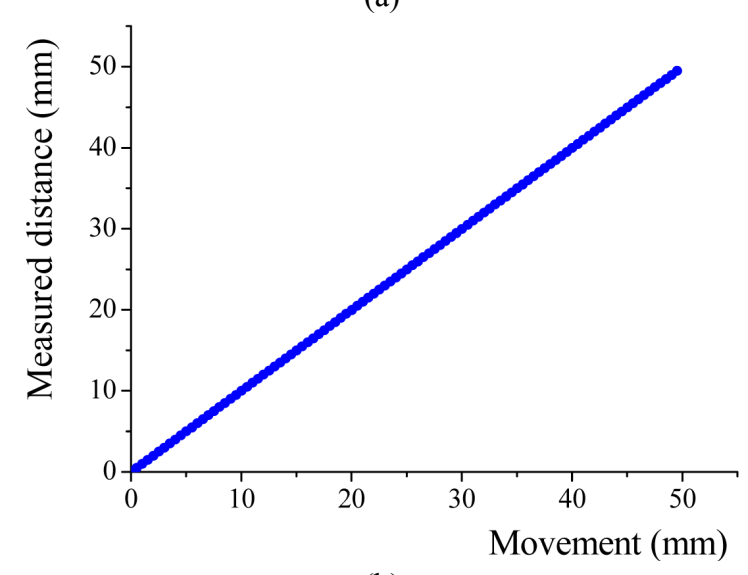

(b)

FIG. 2. Experimental results of combined technique; (a) the black dots are the measured distances which is fitted as saw-wave type with spectrally resolved interferometer and the red dots are calculated distances using Eq. (2), (b) the final distances which is compensated with Eq. (11). with the $13^{\text {th }}$ harmonic of the femtosecond laser repetition frequency $(75.164 \mathrm{MHz})$, which leads to an unambiguity range of $0.154 \mathrm{~m}$. The phase between the reference and measurement waves was measured using a commercial lock-in amplifier (SR830, SRS). In this case, the measurement uncertainty was $0.7 \mathrm{~mm}$ and was mainly dominated by nonlinearity errors and phase measurement errors in the lock in amplifier. The spectrally-resolved interferometer had an unambiguity range of $1.458 \mathrm{~mm}$ which was determined by a solid type Fabry-Perot etalon [7].

For the first time, the measurement results in the spectrally- resolved interferometer were fixed in the form of a saw-tooth wave from the original triangular wave form [7] for simple operations as shown in Fig. 2(a) and the unambiguity range became twice the original one of $2.916 \mathrm{~mm}$. The relative measurement uncertainty was $10^{-5}$ which was mainly caused by the spectrometer. As the results, it was confirmed that the condition in Eq. (12) was satisfied $(0.7 \mathrm{~mm}<1.458 \mathrm{~mm})$. Fig. 2(a) also shows the combined results using Eq. (2). In the results, four points have the wrong $m$ values which results in 2.916 $\mathrm{mm}$ jumps from the right distance.

The first jump point is at $17.526 \mathrm{~mm}$ and the measurement results from the synthetic wavelength interferometer and the spectrally resolved interferometer are $17.54 \mathrm{~mm}$ and $2.884 \mathrm{~mm}$, respectively. The integer count $m$ was calculated as 6 and $\eta$ was 0.084 . The calculated distance was $20.386 \mathrm{~mm}$, which was not in the range of the uncertainty region in the synthetic wavelength interferometer. Thus, $(m+1)$ and $(m-1)$ were checked resulting in a correct integer $m$ of 5 and the final distance was $17.469 \mathrm{~mm}$. Table 1 shows the calculated and compensated distances with the experimental results at the four points. Fig. 2(b) shows the final distance results in the combined technique based on the methods described in the previous section.

On the other hand, another important issue with combining techniques is the total measurement uncertainty. From Eq. (2), the total measurement uncertainty $(\delta D)$ can be expressed as

$$
\begin{aligned}
\delta D & =\sqrt{\left(\frac{\partial D}{\partial m}\right)^{2} \delta m^{2}+\left(\frac{\partial D}{\partial l_{u}}\right)^{2} \delta l_{u}{ }^{2}+\left(\frac{\partial D}{\partial l}\right)^{2} \delta l^{2}} \\
& =\sqrt{l_{u}{ }^{2} \cdot \delta m^{2}+m^{2} \cdot \delta l_{u}{ }^{2}+\delta l^{2}}
\end{aligned}
$$

TABLE 1. Calculated and compensated distances at 4 jump points (mm)

\begin{tabular}{c|c|c|c|c|c}
\hline \hline $\begin{array}{c}\text { Synthetic wavelength } \\
\text { interferometer }\end{array}$ & $\begin{array}{c}\text { Spectrally resolved } \\
\text { interferometer }\end{array}$ & $m$ & $\eta$ & $\begin{array}{c}\text { Calculated } \\
\text { distance }\end{array}$ & $\begin{array}{c}\text { Compensated } \\
\text { distance }\end{array}$ \\
\hline 17.54 & 2.88409 & 6 & 0.084 & 20.38605 & 17.46906 \\
\hline 37.85 & 0.03501 & 12 & 0.981 & 35.03894 & 37.95594 \\
\hline 40.80 & 0.11792 & 13 & 0.993 & 38.03884 & 40.95583 \\
\hline 43.75 & 0.19793 & 14 & 0.999 & 41.03585 & 43.95284 \\
\hline
\end{tabular}


where $\delta m$ is the uncertainty in the selection of $m$ and $\delta l$ is the measurement uncertainty of the FMT. As previously explained, the first term of the right side in Eq. (13) can become zero because $m$ can be calculated and compensated correctly. Consequently, the dominant factor in the total distance measurement uncertainty is from the uncertainty of $l_{u}$ multiplied by a large $m$ value. This uncertainty is akin to the frequency uncertainty in displacement or distance interferometry. Thus, the total uncertainty follows the measurement uncertainty of the FMT, as expected. In addition, the condition in Eq. (12) should be also satisfied for this goal because it shows the relationship between $\mathrm{m}$ and the uncertainty of the RMT to determine $m$. If the measurement uncertainty of the RMT is not enough, $m$ cannot be determined exactly and the total uncertainty can be an order of the unambiguity range of the FMT. Therefore, these conditions should be checked when using the combined method for long distance measurements.

\section{CONCLUSION}

In this paper, we describe the principle using combined techniques to measure distances and define conditions for successful implementation of these techniques. When combining the fine measurement technique (FMT) with the rough measurement technique (RMT), the uncertainty of the RMT should be at least smaller than the half unambiguity range of the FMT. In this experiment, the synthetic wavelength interferometer and spectrally-resolved interferometer were combined to verify the techniques for the proposed conditions. Under these conditions, it was confirmed that the total measurement uncertainty can follow the measurement uncertainty of the FMT as expected.

\section{ACKNOWLEDGMENT}

This research was supported by Basic Science Research Program through the National Research Foundation of Korea (NRF) funded by the Ministry of Education, Science and Technology (2012R1A1A1001842).

\section{REFERENCES}

1. H. Matsumoto, "Synthetic interferometric distance-measuring system using a $\mathrm{CO}_{2}$ laser," Appl. Opt. 25, 493-498 (1986).

2. A. G. Stove, "Linear FMCW radar techniques," IEE Proc. Radar and Signal Processing 139, 343-350 (1992).

3. R. Dändliker, K. Hug, J. Politch, and E. Zimmermann, "High-accuracy distance measurements with multiple-wavelength interferometry," Opt. Eng. 34, 2407-2412 (1995).

4. G. P. Barwood, P. Gill, and R. C. Rowley, "High-accuracy length metrology using multiple-stage swept-frequency interferometry with laser diodes," Meas. Sci. Technol. 9, 10361041 (1998).

5. K. Minoshima and H. Matsumoto, "High-accuracy measurement of 240-m distance in an optical tunnel by use of a compact femtosecond laser," Appl. Opt. 39, 5512-5517 (2000).

6. J. Ye, "Absolute measurement of a long, arbitrary distance to less than an optical fringe," Opt. Lett. 29, 1153-1155 (2004).

7. K.-N. Joo and S.-W. Kim, "Absolute distance measurement by dispersive interferometry using a femtosecond pulse laser," Opt. Express 14, 5954-5960 (2006).

8. Y. Salvadé, N. Schuhler, S. Lévêque, and S. L. Floch, "High-accuracy absolute distance measurement using frequency comb referenced multiwavelength source," Appl. Opt. 47, 2715-2720 (2008).

9. K.-N. Joo, Y. Kim, and S.-W. Kim, "Distance measurements by combined method based on a femtosecond pulse laser," Opt. Express 16, 19799-19806 (2008).

10. I. Coddington, W. C. Swann, L. Nenadovic, and N. R. Newbury, "Rapid and precise absolute distance measurements at long range," Nature Photon. 3, 351-356 (2009).

11. J. Lee, Y.-J. Kim, K. Lee, S. Lee, and S.-W. Kim, "Timeof-flight measurement with femtosecond light pulses," Nature Photon. 4, 716-720 (2010).

12. O. P. Lay, S. Dubovitsky, R. D. Peters, J. P. Burger, S.-W. Ahn, W. H. Steier, H. R. Fetterman, and Y. Chang, "MSTAR: a submicrometer absolute metrology system," Opt. Lett. 28, 890-892 (2003).

13. P. de Groot, "Three-color laser-diode interferometer," Appl. Opt. 30, 3612-3616 (1991). 